\title{
Opportunistic Transmission with Partial Channel Information in Multi-User OFDM Wireless Systems
}

\author{
Jae-Yun Ko and Yong-Hwan Lee \\ School of Electrical Engineering and INMC, Seoul National University \\ Kwanak P. O. Box 34, Seoul, 151-600 Korea
}

\begin{abstract}
In this paper, we consider opportunistic packet scheduling with the use of reduced channel information in multiuser OFDM wireless systems. The amount of channel information can be reduced by exploiting the correlation characteristics in the frequency domain. For a given amount of feedback information, the subcarriers are selected to maximize the system capacity. A simplified algorithm is proposed for the selection of subcarriers, making the proposed scheme realizable. Simulation results show that the proposed scheme provides significant performance improvement over conventional schemes especially when the channel is highly correlated in the frequency domain and/or the amount of allowable feedback information is small.
\end{abstract}

\section{INTRODUCTION}

Opportunistic scheduling is one of the major transmission techniques indispensable for the improvement of spectral efficiency in a packet-based multi-user wireless system [1], [2]. It enables to achieve so-called multi-user diversity (MUD) gain by exploiting the fading characteristics of each user. When a multi-user OFDM system employs an opportunistic scheduling scheme, it may encounter implementation problems due to heavy feedback signaling burden in the uplink. To alleviate this problem, recent research has considered the use of reduced channel information [3]-[6].

To reduce the channel information, the use of a channel clustering technique was considered in OFDM based multi-user systems [3], where the receiver reports the information of subchannels each of which comprises several subcarriers. The amount of feedback information can be reduced by allowing each user to report only good $M$ subchannels instead of all subchannels [4]. The use of a threshold method was considered to reduce the feedback overhead [5]. This idea was further simplified to the use of one bit channel information indicating whether the subchannel quality is good or not for data transmission [6]. The ideas in [5] and [6] can easily be applied to a system with parallel multiple channels such as OFDM systems. However, these schemes can provide a desired performance only when the number of users is sufficiently large (e.g., several hundreds of users in [6] and near one hundred users in [4]), making it impractical in real operating environments.

We consider the reduction of feedback information exploiting the channel correlation characteristics. Since adjacent subcarriers are more likely to be correlated with each other, it may be possible to extract the whole channel information from partial one. The base station (BS) receives the channel information of a small number of subcarriers from users and then estimates the channel information of the rest of

This work was in part supported by Samsung Electronics research project (Skypass4G) subcarriers from the reported channel information by making the use of the channel correlation properties. Subcarriers can be selected to maximize the expected system capacity depending on the number of users, the amount of feedback information and the channel correlation characteristics.

Following introduction, Section II describes the system model in consideration. The proposed scheme is presented in Section III and IV: we consider the use of the frequency correlation properties for the user selection in Section III and the selection of feedback channel is presented in Section IV. The performance of the proposed scheme is compared with previous ones in Section V. Finally, conclusions are summarized in Section VI.

\section{SySTEM MOdEL}

We consider the downlink of an OFDM based multi-user wireless system servicing $K$ active users. For ease of description, we assume that users have the same average signal-to-noise power ratio (SNR). ${ }^{1}$ We also assume the transmission over a wireless channel whose impulse response is described by a finite impulse response filter with $L$ taps. The $l$-th $(l=1, \ldots, L)$ propagation path of user $k$ can be represented in terms of delay $\tau_{k, l}$ and complex-valued fading gain $h_{k, l}$. We assume that all the path gains are independent complex Gaussian random variables with zero mean and variance $\sigma_{k, l}^{2}$, and that the channel gains are normalized such that $\sum_{l=1}^{L} \sigma_{k, l}^{2}=1$. By adding a cyclic prefix having a duration longer than $\tau_{k, L}$, the OFDM system with $N$ subcarriers converts a frequency selective fading channel into $N$ parallel channels with flat fading [8]. Thus, user $k$ has a channel gain $g_{k}(n)$ at the $n$-th subcarrier given by

$$
g_{k}(n)=\sum_{l=1}^{L} h_{k, l} \exp \left(-\frac{j 2 \pi n \tau_{k, l}}{N T_{s}}\right), \quad n=1, \ldots, N,
$$

where $T_{s}$ is the sampling interval. Note that $g_{k}(n)$ is a zero mean complex Gaussian random variable with unit variance where $n=1, \ldots, N$.

We define the channel correlation between the subcarriers by

$$
R_{k}(\Delta n)=E\left[g_{k}^{*}(n) g_{k}(n+\Delta n)\right]=\sum_{l=1}^{L} \sigma_{k, l}^{2} \exp \left(-\frac{j 2 \pi \Delta n \tau_{k, l}}{N T_{s}}\right),
$$

where $E[x]$ denotes the expectation of random variable $x$ and the superscript $*$ denotes the complex conjugate. Assuming that the average SNR is equal to $\gamma_{o}$, the instantaneous SNR of

\footnotetext{
${ }^{1}$ When users have different SNRs, the proposed scheme can be employed with the use of channel gain normalized with respect to the average SNR.
} 
subcarrier $n$ for user $k$ is given by $\eta_{k}(n)=\left|g_{k}(n)\right|^{2} \gamma_{o}$. Then the autocorrelation of the SNR between the subcarriers is represented as

$$
E\left[\eta_{k}(n) \eta_{k}(n+\Delta n)\right]=\gamma_{o}^{2} E\left[\left|g_{k}(n)\right|^{2}\left|g_{k}(n+\Delta n)\right|^{2}\right] .
$$

By using that $E\left[|x|^{2}|y|^{2}\right]=E\left[|x|^{2}\right] E\left[|y|^{2}\right]+\left|E\left[x^{*} y\right]\right|^{2}$, where $x$ and $y$ are zero-mean complex Gaussian variables, (3) can be rewritten as

$$
\begin{aligned}
E\left[\eta_{k}(n) \eta_{k}(n+\Delta n)\right]= & \gamma_{o}^{2} E\left[\left|g_{k}(n)\right|^{2}\right] E\left[\left|g_{k}(n+\Delta n)\right|^{2}\right] \\
& +\gamma_{o}^{2}\left|E\left[g_{k}^{*}(n) g_{k}(n+\Delta n)\right]\right|^{2} \\
= & \gamma_{o}^{2}\left(1+\left|R_{k}(\Delta n)\right|^{2}\right) .
\end{aligned}
$$

It can be seen that as the channel correlation between the subcarriers increases, so does the SNR correlation between the subcarriers. This implies that it may be possible to get the whole SNR information from partial one.

In this paper, we assume that users can estimate the channel information by making the use of common pilot signal and the BS can get the channel information from users through a feedback channel in the uplink. We also assume that the statistical channel information such as the correlation between the subcarriers can be delivered reliably to the BS via a low rate feedback channel in the uplink. Note that the correlation between the subcarriers can also be obtained from the power delay profile of channel, which may require much less feedback information especially when the number of subcarriers is large and/or the delay spread of the channel is small. Besides, when the carrier frequencies of the uplink and the downlink have a small deviation, the correlation estimated in the uplink can be applied to the downlink because the average channel characteristic is not much different [7].

\section{Opportunistic Scheduling with Partial ChanNel INFORMATION}

Consider the case when user $k$ estimates the SNR of all $N$ subcarriers and reports the SNR of $M(\leq N)$ subcarriers. Letting $\left\{\psi_{k, 1}, \ldots, \psi_{k, M}\right\}$ be the index of the corresponding subcarriers, the SNRs reported by user $k$ can be represented as $\Omega_{k}=\left\{\eta_{k}\left(\psi_{k, 1}\right), \ldots, \eta_{k}\left(\psi_{k, M}\right)\right\}$. Then we consider packet scheduling in the BS to maximize the system capacity for a given set $\left\{\Omega_{1}, \ldots, \Omega_{K}\right\}$. To this end, we need to optimally find the corresponding $M$ subcarriers.

The scheduler can maximize the system capacity by choosing a user in the best SNR condition for each subcarrier. In a conventional scheduling scheme, users who report the SNR are only considered for the scheduling [3], [4]. Moreover, the scheduler allocates subcarriers with no SNR information to users in a random manner [4] or does not schedule them at all [3]. This issue can be alleviated by exploiting the channel correlation.

It is possible to describe jointly Gaussian random variables in terms of their first and second order statistics [9]. Thus, $g_{k}(n)$ can be expressed in terms of $g_{k}\left(n^{\prime}\right)$ as

$$
g_{k}(n)=R_{k}\left(\left|n-n^{\prime}\right|\right) g_{k}\left(n^{\prime}\right)+\sqrt{1-\left|R_{k}\left(\left|n-n^{\prime}\right|\right)\right|^{2}} z
$$

where $z$ is a zero mean complex Gaussian random variable with unit variance and independent of $g_{k}\left(n^{\prime}\right)$. Then, subcarrier $n$ has an SNR represented as

$$
\begin{aligned}
& \eta_{k}(n)=\gamma_{o}\left|g_{k}(n)\right|^{2} \\
& \quad=\left|R_{k}\left(\left|n-n^{\prime}\right|\right)\right|^{2} \eta_{k}\left(n^{\prime}\right)+\left(1-\left|R_{k}\left(\left|n-n^{\prime}\right|\right)\right|^{2}\right)|z|^{2} \gamma_{o}+C,
\end{aligned}
$$

where $C$ is a random variable with zero mean. Thus, for a given $\Omega_{k}$, the BS can estimate the SNR of all subcarriers as ${ }^{2}$

$$
\begin{aligned}
\tilde{\eta}_{k}(n) & =E\left[\eta_{k}(n) \mid \eta_{k}\left(\lambda_{k, n}\right)\right] \\
& =\left|R_{k}\left(\left|n-\lambda_{k, n}\right|\right)\right|^{2} \eta_{k}\left(\lambda_{k, n}\right)+\left(1-\left|R_{k}\left(\left|n-\lambda_{k, n}\right|\right)\right|^{2}\right) \gamma_{o}
\end{aligned}
$$

where

$$
\lambda_{k, n}=\arg \max _{i=\left\{\psi_{k, 1}, \ldots, \psi_{k, M}\right\}}\left|R_{k}(|n-i|)\right| .
$$

The scheduler can allocate each subcarrier to a user having the maximum expected SNR. Letting $\pi_{n}$ be the index of the user assigned to subcarrier $n$ i.e.,

$$
\pi_{n}=\arg \max _{k} \tilde{\eta}_{k}(n)
$$

the overall capacity of the proposed scheme is given by

$$
C_{\text {prop }}=\sum_{n=1}^{N} E\left[B_{\Delta} \log _{2}\left(1+\eta_{\pi_{n}}(n)\right)\right]
$$

where $B_{\Delta}$ is the bandwidth of the subcarrier. For simplicity of description, we assume that $B_{\Delta}=1$.

To verify the validity of the proposed scheme, consider a simple case where each user reports the SNR of the same $M$ subcarriers $\left\{\psi_{1}, \ldots, \psi_{M}\right\}$. Letting $\Gamma(n)$ be the maximum SNR of the $n$-th subcarrier, where $n \in\left\{\psi_{1}, \ldots, \psi_{M}\right\}$,

$$
\Gamma(n)=\max _{k} \eta_{k}(n),
$$

the capacity of subcarriers with reported SNR can be represented as

$$
\Lambda_{\mathrm{w}}=E\left[\log _{2}(1+\Gamma(n))\right]
$$

Since $\Gamma(n)$ is the maximum value of $K$ independent exponential random variables, (12) can be expressed as [10]

$$
\Lambda_{\mathrm{w}}=\frac{K}{\ln 2} \sum_{k=0}^{K-1}\left(\begin{array}{c}
K-1 \\
k
\end{array}\right)(-1)^{k} \exp \left(\frac{k+1}{\gamma_{0}}\right) \frac{E i\left((k+1) / \gamma_{0}\right)}{k+1},
$$

\footnotetext{
${ }^{2}$ More intelligent estimation methods with higher complexity can be used, e.g., one that considers all subcarriers for which feedback is provided. But the simulation results indicate that this simple estimation method still provides a desirable performance.
} 
where $\operatorname{Ei}(z)=\int^{\infty} e^{-t} / t d t$. Since the expected value of $\Gamma(n)$ can be expressed by [11]

$$
E[\Gamma(n)]=\sum_{k=1}^{K} \frac{1}{k},
$$

it can be shown that

$$
\begin{aligned}
\Lambda_{\mathrm{w}} & \leq \log _{2}\left(1+\gamma_{o} E[\Gamma(n)]\right) \\
& =\log _{2}\left(1+\gamma_{o} \sum_{k=1}^{K} \frac{1}{k}\right) .
\end{aligned}
$$

Conventional schedulers allocate subcarriers with no channel information to users in a random manner. Since this can be considered as a special case of (13) with $K=1$, the capacity of these subcarriers can be represented as

$$
\Lambda_{\mathrm{wo}} \leq \log _{2}\left(1+\gamma_{o}\right) .
$$

It can be shown that the overall capacity of the conventional scheduler is bounded by

$$
C_{\text {conv }} \leq M \log _{2}\left(1+\gamma_{o} \sum_{k=1}^{K} \frac{1}{k}\right)+(N-M) \log _{2}\left(1+\gamma_{o}\right) .
$$

On the other hand, the proposed scheduler can allocate subcarriers to users as if it has full SNR information. Since the maximum value of the estimated SNR can be represented as

$$
\begin{aligned}
\tilde{\Gamma}(n) & =\max _{k} \tilde{\eta}_{k}(n) \\
& =\left|R_{\pi_{n}}\left(\left|n-\lambda_{\pi_{n}, n}\right|\right)\right|^{2} \Gamma_{K}+\left(1-\left|R_{\pi_{n}}\left(\left|n-\lambda_{\pi_{n}, n}\right|\right)\right|^{2}\right) \gamma_{o},
\end{aligned}
$$

the capacity of these subcarriers is bounded by

$$
\begin{aligned}
\Lambda_{\text {est }}(n) & \leq \log _{2}(1+\tilde{\Gamma}(n)) \\
& =\log _{2}\left(1+\gamma_{o}\left(1+\left|R_{\pi_{n}}\left(\left|n-\lambda_{\pi_{n}, n}\right|\right)\right|^{2} \sum_{k=2}^{K} \frac{1}{k}\right)\right) .
\end{aligned}
$$

Thus, it can be shown that the overall capacity of the proposed scheme is bounded by

$$
\begin{aligned}
C_{\text {prop }} \leq & M \log _{2}\left(1+\gamma_{o} \sum_{k=1}^{K} \frac{1}{k}\right) \\
& +\sum_{\substack{n=1 \\
n \in\left\{\psi_{1}, \ldots, \psi_{M}\right\}}}^{N} \log _{2}\left(1+\gamma_{o}\left(1+\left|R_{\pi_{n}}\left(\left|n-\lambda_{\pi_{n}, n}\right|\right)\right|^{2} \sum_{k=2}^{K} \frac{1}{k}\right)\right) .
\end{aligned}
$$

Compared to the conventional scheme, the proposed scheme has a capacity improvement of

$$
\begin{aligned}
C_{\text {gain }} & =C_{\text {prop }}-C_{\text {conv }} \\
& \approx \sum_{\substack{n=1 \\
n \notin\left\{\psi_{1}, \ldots, \psi_{M}\right\}}}^{N} \log _{2}\left(\frac{1+\gamma_{o}\left(1+\left|R_{\pi_{n}}\left(\left|n-\lambda_{\pi_{n}, n}\right|\right)\right|^{2} \sum_{k=2}^{K} 1 / k\right)}{1+\gamma_{o}}\right) .
\end{aligned}
$$

The capacity gain increases as the correlation between the subcarriers increases. On the other hand, compared to the use of full SNR information whose capacity is given by

$$
C_{\text {full }}=\sum_{n=1}^{N} \log _{2}\left(1+\gamma_{o} \sum_{k=1}^{K} \frac{1}{k}\right),
$$

the proposed scheme has a capacity loss given by

$$
\begin{gathered}
C_{\text {loss }}=C_{\text {full }}-C_{\text {prop }} \\
\approx \sum_{\substack{n=1 \\
n \in\left\{\psi_{1}, \ldots, \psi_{M}\right\}}}^{N} \log _{2}\left(\frac{1+\gamma_{o} \sum_{k=1}^{K} 1 / k}{1+\gamma_{o}\left(1+\left|R_{\pi_{n}}\left(\left|n-\lambda_{\pi_{n}, n}\right|\right)\right|^{2} \sum_{k=2}^{K} 1 / k\right)}\right) .
\end{gathered}
$$

It can be seen that the capacity loss is a decreasing function of $\left|R_{\pi_{n}}\left(\left|n-\lambda_{\pi_{n}, n}\right|\right)\right|$. Thus, to minimize the performance loss, we need to find the optimum information set $\Omega_{k}$ for each user that maximizes the correlation values for a given amount of feedback information. Note that the upper bound approximation of the proposed scheme is tighter than that of the conventional scheme due to larger MUD gain [12] and thus the actual capacity gain is somewhat larger than (21). Similarly, the actual capacity loss is somewhat larger than (23).

\section{SELECTION OF SUBCARRIERS FOR SNR REPORTING}

We assume that each user reports the channel information independent of each other. Let $Q_{k, n}$ be the index of the user having the highest SNR of subcarrier $n$ among $K$ users excluding user $k$, defined by

$$
Q_{k, n}=\arg \max _{i \neq k} \tilde{\eta}_{i}(n) .
$$

Then, the instantaneous SNR and its estimated value can be represented as $\eta_{Q_{k, n}}(n)$ and $\tilde{\eta}_{Q_{k, n}}(n)$, respectively.

Let $\tilde{\eta}_{k}\left(n \mid \Omega_{k}\right)$ be the estimated SNR of subcarrier $n$ for user $k$ for a given partial SNR information $\Omega_{k}$. If $\tilde{\eta}_{k}\left(n \mid \Omega_{k}\right)$ is larger than $\tilde{\eta}_{Q_{k, n}}(n)$, the scheduler allocates subcarrier $n$ to user $k$, yielding an expected capacity of $\log _{2}\left(1+\eta_{k}(n)\right)$. On the other hand, if not, the scheduling result is unchanged and thus the capacity of subcarrier $n$ is $\log _{2}\left(1+\eta_{Q_{k, n}}(n)\right)$. Thus, the corresponding capacity can be represented as

$$
\begin{aligned}
\Lambda_{\Omega_{k}}(n)= & \int_{\tilde{\eta}_{k}\left(n \mid \Omega_{k}\right)}^{\infty} \log _{2}\left(1+\eta_{Q_{k, n}}(n)\right) f_{\eta}(x) d x \\
& +\int_{0}^{\tilde{\eta}_{k}\left(n \mid \Omega_{k}\right)} \log _{2}\left(1+\eta_{k}(n)\right) f_{\eta}(x) d x,
\end{aligned}
$$

where $f_{\eta}(x)$ is the probability density function $(p d f)$ of $\tilde{\eta}_{Q_{k, n}}(n)$ which needs to be estimated at the BS and then to be informed to the user. Since $\eta_{Q_{k, n}}(n)$ is not available to user $k$, (25) can be approximated by assuming $\eta_{Q_{k, n}}(n) \approx \tilde{\eta}_{Q_{k, n}}(n)$, i.e.,

$$
\begin{aligned}
\Lambda_{\Omega_{\Omega_{k}}}(n) \approx & \int_{\tilde{\eta}_{k}\left(n \mid \Omega_{\Omega_{k}}\right)}^{\infty} \log _{2}(1+x) f_{\eta}(x) d x \\
& +\int_{0}^{\tilde{\eta}_{k}\left(n \mid \Omega_{k}\right)} \log _{2}\left(1+\eta_{k}(n)\right) f_{\eta}(x) d x .
\end{aligned}
$$

When no SNR information is reported from user $k$, the estimated SNR of all subcarriers is set to an average SNR of 
the user, i.e., $\tilde{\eta}_{k}(n \mid \varnothing)=\gamma_{o}$ for $n=1, \ldots, N$, where $\varnothing$ denotes no channel information. Then the corresponding capacity is given by

$$
\Lambda_{\phi}(n) \approx \int_{\gamma_{o}}^{\infty} \log _{2}(1+x) f_{\eta}(x) d x+\int_{0}^{\gamma_{o}} \log _{2}\left(1+\eta_{k}(n)\right) f_{\eta}(x) d x .
$$

The capacity improvement due to the use of feedback information $\Omega_{k}$ can be approximated by

$$
\begin{aligned}
& \Delta_{\Omega_{k}}=\sum_{n=1}^{N}\left(\Lambda_{\Omega_{k}}(n)-\Lambda_{\phi}(n)\right) \\
& \approx \sum_{n=1}^{N}\left(\int_{\gamma_{o}}^{\tilde{\eta}_{k}\left(n \mid \Omega_{k}\right)}\left(\log _{2}\left(1+\eta_{k}(n)\right)-\log _{2}(1+x)\right) f_{\eta}(x) d x\right) .
\end{aligned}
$$

The main concern is to find an optimum set $\Omega_{k}$ that maximizes $\Delta_{\Omega_{i}}$. It is possible to find an optimum set by exhaustively searching for all possible cases (e.g., $N !((N-M) ! M !)^{-1}$ cases in the case of reporting $M$ SNRs among $N$ SNRs). The complexity of this searching process increases exponentially in proportion to $N$ and/or $M$, making it impractical for the implementation.

To make the implementation practical, we consider a simple search process where the reporting subcarriers are sequentially determined by disregarding subcarriers highly correlated with subcarriers previously selected. As a result, the reported information $\Omega_{k}$ does not contain SNRs of subcarriers which are highly correlated to each other. The set $\Omega_{k}$ of user $k$ can be determined as follows:

Step I: Initialize

$$
\Theta_{1}=\{1,2, \ldots, N\}, \Omega_{k}=\varnothing \text { and } i=1 .
$$

Step II: Select the reporting subcarrier, and then update the feedback information and candidate subcarrier set as

$$
\begin{aligned}
& \psi_{i}=\arg \max _{n \in \Theta_{i}}\left\{\eta_{k}(n)\right\}, \Omega_{k} \leftarrow \Omega_{k} \cup \eta_{k}\left(\psi_{i}\right) \text { and } \\
& \Theta_{i+1}=\left\{n \in \Theta_{i}|| R_{k}\left(\left|n-\psi_{i}\right|\right) \mid<\delta_{k}\right\} .
\end{aligned}
$$

Step III: If $\left|\Omega_{k}\right|<M$ and $\Theta_{i+1} \neq \varnothing$, then $i \leftarrow i+1$ and go to Step II. Else stop.

Here $\delta_{k}$ is a threshold value which needs to be optimized according to the operating environment, such as the frequency selectivity of channel, the number of users and the amount of feedback information. Although the optimum threshold value is yet to be determined analytically, it can easily be determined by experiments in practice.

\section{Performance Evaluation}

The performance of the proposed scheme is verified by computer simulation. The simulation environment is almost identical to [13] as summarized in Table I, where the whole 2048 subcarriers are divided into 256 subchannels. The SNR of each subchannel is obtained by averaging the SNR of 8 subcarriers. We assume that the estimation of SNR is perfect and that the channel information is delivered without delay and
TABLE I

SIMULATION CONDITION

\begin{tabular}{|l|l|}
\hline Number of subcarriers & 2048 \\
\hline Number of subchannels & 256 \\
\hline Average SNR & $10 \mathrm{~dB}$ \\
\hline rms delay spread & $100 \mathrm{nsec}$ \\
\hline Channel estimation & Ideal \\
\hline
\end{tabular}

error to clearly examine the effect of partial channel

information on the system performance. We also assume that the channel has an exponentially decaying power delay profile with $100 \mathrm{nsec} r m s$ delay spread at an average SNR of $10 \mathrm{~dB}$, unless explicitly stated otherwise. For comparison, we consider three conventional schemes, called best-M feedback (referred to Best-M) [6], one-bit feedback (referred to One-Bit) [5] and full feedback (referred to Full). In the Best-M scheme, each user reports $M$ SNRs corresponding to $M$ best subchannels. The scheduler assigns subchannels with reported SNR information to users in a scheduled manner and assigns subchannels with no SNR information to users in a random manner. We assume that the SNR is reported in a form of 5 bits (i.e., from $-3 \mathrm{~dB}$ to $28 \mathrm{~dB}$ with a resolution of $1 \mathrm{~dB}$ ). In the One-Bit scheme, each user reports the SNR of each subchannel in a single bit form. For the simulation, the threshold value for the SNR quantization is optimized by searching for each environment. In the Full scheme, each user reports the SNR of all subchannels with full precision. This scheme is treated as a reference. In the proposed scheme, each user reports the SNR of $M$ subchannels in a form of 5-bit as in the Best-M, where $M$ subchannels are determined using the proposed algorithm with $\delta_{k}=0.6$ for all users.

Fig. 1 depicts the performance associated with the number $(M)$ of reported SNRs when $K=8$. For fair comparison, all the schemes except the Full scheme use the same amount of feedback signal. The proposed scheme and One-Bit scheme respectively require $\log _{2}(256)+5$ and $\log _{2}(256)+1$ bits for the SNR reporting of each subchannel, where the first and second terms are for the representation of subchannel index and SNR value, respectively. The number $\left(M^{\prime}\right)$ of reported subcarriers in the One-Bit scheme is determined by

$$
M^{\prime}=\left\lceil\frac{\log _{2}(256)+5}{\log _{2}(256)+1} M\right\rceil
$$

where $\lceil x\rceil$ denotes the smallest integer number larger than or equal to $x$. It can be seen that the proposed scheme provides performance almost $90 \%$ of the Full scheme only with the use of 8 reported SNRs, considerably outperforming other schemes. This suggests the validity of the proposed algorithm. It can also be seen that the One-Bit scheme outperforms the Best-M scheme when the same number of feedback bits are used. This suggests that the use of more channel information with less accuracy can yield better performance than the use of less channel information with better accuracy, assuming the use of the same amount of information bits. 


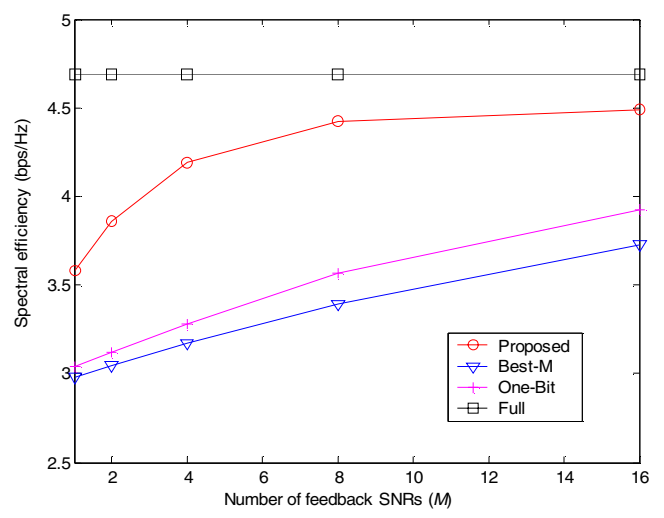

Fig. 1. Performance associated with the number of feedback SNRs when $K=8$.

Fig. 2 depicts the performance of the proposed scheme in terms of the number of users when $M=4$. For fair comparison, the One-Bit scheme uses $M^{\prime}=6(=\lceil 5.778\rceil)$. It can be seen that the proposed scheme significantly outperforms other schemes using the same amount of feedback information. It can also be seen that although the performance of all the schemes approaches to that of the Full scheme as the number of users increases [4], [6], the proposed scheme fast approaches to the Full scheme compared to other schemes.

Fig. 3 shows the effect of the frequency selectivity on the system capacity. It can be seen that the performance of the proposed scheme is somewhat more affected than others as the delay spread of the channel increases (or equivalently the selectivity of the channel increases). This is mainly due to the fact that subchannels are less correlated to each other as the frequency selectivity increases. On the other hand, unless the frequency selectivity is too high, the proposed scheme significantly outperforms other schemes. It can also be seen that, unlike the proposed scheme, other schemes provide slight performance improvement as the frequency selectivity increases. This is due to that as the frequency selectivity of channel increases, other schemes can increase the selection diversity by selecting good subchannels from a larger number of independent subchannels.

\section{CONCLUSIONS}

We have considered the use of partial SNR information for opportunistic transmission in an OFDM based multi-user wireless system. By exploiting the channel correlation characteristics, the proposed scheme can reduce the feedback signaling burden for the channel information, while minimizing the performance loss due to reduced channel information. To make the proposed algorithm realizable, we have proposed a simplified algorithm that sequentially determines the subcarriers based on a single threshold. The simulation results show the superiority of the proposed scheme over the conventional ones especially when the channel correlation in frequency domain is high and/or the amount of feedback information is small.

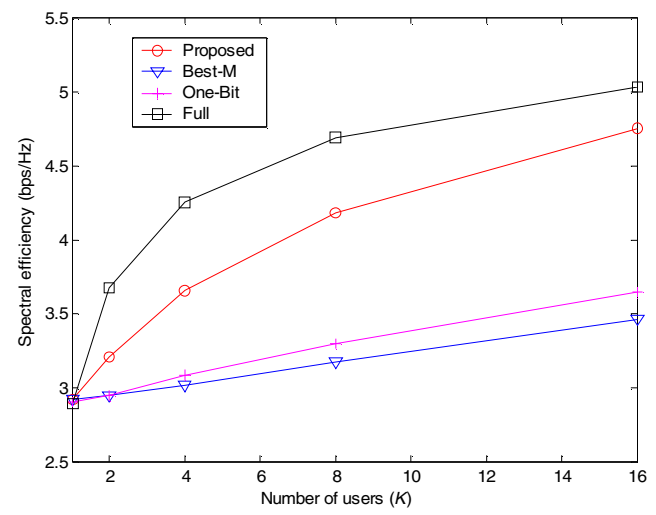

Fig. 2. Performance associated with the number of users when $M=4$.

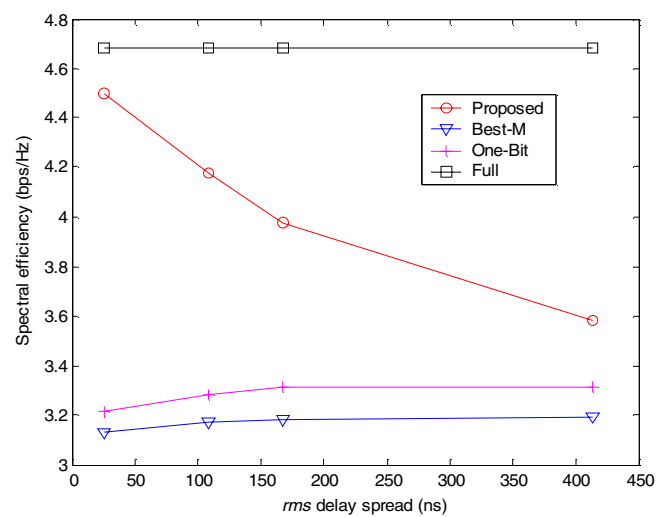

Fig. 3. Performance according to the delay spread when $M=4$ and $K=8$.

\section{REFERENCES}

[1] X. Liu, E. K. P. Chong and N. B. Shroff, "A Framework for Opportunistic Scheduling in Wireless Networks," Computer Networks, vol. 41, pp. 451474, Mar. 2003.

[2] P. Viswanath, D. N. C. Tse and R. Laroia, "Opportunistic Beamforming Using Dumb Antennas," IEEE Trans. Inform. Theory, vol. 48, no 6, pp. 1277-1294, June 2002.

[3] P. Svedman, S. Wilson, L. Cimini and B. Ottersted, "A Simplified Opportunistic Feedback and Scheduling Scheme for OFDM," in Proc. IEEE Veh. Technol. Conf., pp. 1878-1882, May 2004.

[4] Z.-H. Han and Y.-H. Lee, "Opportunistic Scheduling with Partial Channel Information in OFDMA/FDD Systems," in Proc. IEEE Veh. Technol. Conf., pp. 511-514, Sept. 2004.

[5] D. Gesbert and M. Alouini, "How Much Feedback is Multi-User Diversity Really Worth?," in Proc. IEEE Int. Conf. Commun., pp. 234-238, June 2004.

[6] S. Sanayei and A. Nosratinia, "Exploiting Multiuser Diversity with Only 1-bit Feedback," in Proc. IEEE Wireless Commun. and Networking Conf., pp. 978-983, Mar. 2005.

[7] G. Barriac and U. Madhow, "Space-Time Precoding for Mean and Covariance Feedback: Application to Wideband OFDM," IEEE Trans. Commun., vol. 54, no. 1, pp. 96-107, Jan. 2006.

[8] R. Van Nee and R. Prasad, OFDM for Wireless Multimedia Communications, Artech House Publishers, 2000.

[9] J. G. Proakis, Digital Communications, McGraw-Hill, 4-th ed., 2001.

[10] M. S. Alouini and A. J. Goldsmith, "Capacity of Rayleigh Fading Channels under Different Adaptive Transmission and DiversityCombining Techniques," IEEE Trans. Vehicular Tech., vol. 48, no. 4, pp. 1165-1181, July 1999.

[11] G. L. Stuber, Principles of Mobile Communication, Kluwer Academic Publishers, 1996.

[12] S. Sanayei, A. Nosratinia and N. Aldhahir, "Opportunistic Dynamic Subchannel Allocation in Multiuser OFDM Networks with Limited Feedback," in Proc. IEEE Information Theory Workshop 2004, pp. 182 186, Oct. 2004.

[13] J. Moon, J.-Y. Ko and Y.-H. Lee, "A Framework Design for the NextGeneration Radio Access System," IEEE J. Select. Areas Commun., vol. 24, no. 3, pp. 554-564, Mar. 2006. 